\title{
Common fixed point results for weak contractive mappings in ordered $b$-dislocated metric spaces with applications
}

\author{
Nawab Hussain', Jamal Rezaei Roshan ${ }^{2 *}$, Vahid Parvaneh ${ }^{3}$ and Mujahid Abbas ${ }^{4}$
}

\section{"Correspondence:}

jmlroshan@gmail.com

${ }^{2}$ Department of Mathematics,

Qaemshahr Branch, Islamic Azad

University, Qaemshahr, Iran

Full list of author information is

available at the end of the article

\begin{abstract}
We first introduce a new concept of $b$-dislocated metric space as a generalization of dislocated metric space and analyze different properties of such spaces.

A fundamental result for the convergence of sequences in $b$-dislocated metric spaces is established and is employed to prove some common fixed point results for four mappings satisfying the generalized weak contractive condition in partially ordered b-dislocated metric spaces. Moreover, some examples and applications to integral equations are given here to illustrate the usability of the obtained results.
\end{abstract}

MSC: Primary $47 \mathrm{H} 10$; secondary $54 \mathrm{H} 25$

Keywords: coincidence point; common fixed point; dislocated metric space; $b$-dislocated metric space; dominating and dominated maps; altering distance function

\section{Introduction and preliminaries}

The Banach contraction principle is one of the simplest and most applicable results of metric fixed point theory. It is a popular tool for proving the existence of solution of problems in different fields of mathematics. There are several generalizations of the Banach contraction principle in literature on metric fixed point theory [1-10]. Hitzler and Seda [11] introduced the concept of dislocated topologies and named their corresponding generalized metric a dislocated metric. They have also established a fixed point theorem in complete dislocated metric spaces to generalize the celebrated Banach contraction principle. The notion of dislocated topologies has useful applications in the context of logic programming semantics (see [12]). Further useful results can be seen in [13-23].

Definition 1.1 [11] Let $X$ be a nonempty set. A mapping $d_{l}: X \times X \rightarrow[0, \infty)$ is called a dislocated metric (or simply $d_{l}$-metric) if the following conditions hold for any $x, y, z \in X$ :

(i) If $d_{l}(x, y)=0$, then $x=y$;

(ii) $d_{l}(x, y)=d_{l}(y, x)$;

(iii) $d_{l}(x, y) \leq d_{l}(x, z)+d_{l}(z, y)$.

The pair $\left(X, d_{l}\right)$ is called a dislocated metric space or a $d_{l}$-metric space. Note that when $x=y, d_{l}(x, y)$ may not be 0 .

Example 1.2 If $X=R^{+} \cup\{0\}$, then $d_{l}(x, y)=x+y$ defines a dislocated metric on $X$.

\section{Springer}

(C2013 Hussain et al: licensee Springer. This is an Open Access article distributed under the terms of the Creative Commons Attribution License (http://creativecommons.org/licenses/by/2.0), which permits unrestricted use, distribution, and reproduction in any medium, provided the original work is properly cited. 
Definition 1.3 [11] A sequence $\left\{x_{n}\right\}$ in a $d_{l}$-metric space is called: (1) a Cauchy sequence if, given $\varepsilon>0$, there exists $n_{0} \in \mathbb{N}$ such that for all $n, m \geq n_{0}$, we have $d_{l}\left(x_{m}, x_{n}\right)<\varepsilon$ or $\lim _{n, m \rightarrow \infty} d_{l}\left(x_{n}, x_{m}\right)=0$, (2) convergent with respect to $d_{l}$ if there exists $x \in X$ such that $d_{l}\left(x_{n}, x\right) \rightarrow 0$ as $n \rightarrow \infty$. In this case, $x$ is called the limit of $\left\{x_{n}\right\}$ and we write $x_{n} \rightarrow x$.

A $d_{l}$-metric space $X$ is called complete if every Cauchy sequence in $X$ converges to a point in $X$.

Definition 1.4 A nonempty set $X$ is called an ordered dislocated metric space if it is equipped with a partial ordering $\preceq$ and there exists a dislocated metric $d_{l}$ on $X$.

Definition 1.5 Let $(X, \preceq)$ be a partially ordered set. Then $x, y \in X$ are called comparable if $x \preceq y$ or $y \preceq x$ holds.

Definition 1.6 [1] Let $(X, \preceq)$ be a partially ordered set. A self-mapping $f$ on $X$ is called dominating if $x \preceq f x$ for each $x$ in $X$.

Example 1.7 [1] Let $X=[0,1]$ be endowed with the usual ordering, and let $f: X \rightarrow X$ be defined by $f x=\sqrt[n]{x}$. Since $x \leq x^{\frac{1}{n}}=f x$ for all $x \in X$, therefore $f$ is a dominating map.

Definition 1.8 [1] Let $(X, \preceq)$ be a partially ordered set. A self-mapping $f$ on $X$ is called dominated if $f x \preceq x$ for each $x$ in $X$.

Example 1.9 [1] Let $X=[0,1]$ be endowed with the usual ordering, and let $f: X \rightarrow X$ be defined by $f x=x^{n}$ for some $n \in \mathbb{N}$. Since $f x=x^{n} \leq x$ for all $x \in X$, therefore $f$ is a dominated map.

In the following, we give the definition of a $b$-dislocated metric space.

Definition 1.10 Let $X$ be a nonempty set. A mapping $b_{d}: X \times X \rightarrow[0, \infty)$ is called a $b$-dislocated metric (or simply $b_{d}$-metric) if the following conditions hold for any $x, y, z \in X$ and $s \geq 1$ :

$\left(\mathrm{b}_{\mathrm{d} 1}\right)$ If $b_{d}(x, y)=0$, then $x=y$;

$\left(\mathrm{b}_{\mathrm{d} 2}\right) b_{d}(x, y)=b_{d}(y, x)$;

$\left(\mathrm{b}_{\mathrm{d} 3}\right) b_{d}(x, y) \leq s\left(b_{d}(x, z)+b_{d}(z, y)\right)$.

The pair $\left(X, b_{d}\right)$ is called a $b$-dislocated metric space or a $b_{d}$-metric space. It should be noted that the class of $b_{d}$-metric spaces is effectively larger than that of $d_{l}$-metric spaces, since a $b_{d}$-metric is a $b_{l}$-metric when $s=1$.

Here, we present an example to show that in general a $b$-dislocated metric need not be a $b_{l}$-metric.

Example 1.11 Let $\left(X, d_{l}\right)$ be a dislocated metric space, and $b_{d}(x, y)=\left(b_{l}(x, y)\right)^{p}$, where $p>1$ is a real number. We show that $b_{d}$ is a $b$-dislocated metric with $s=2^{p-1}$.

Obviously, conditions $\left(\mathrm{b}_{\mathrm{d} 1}\right)$ and $\left(\mathrm{b}_{\mathrm{d} 2}\right)$ of Definition 1.10 are satisfied. 
If $1<p<\infty$, then the convexity of the function $f(x)=x^{p}(x>0)$ implies that $\left(\frac{a+b}{2}\right)^{p} \leq$ $\frac{1}{2}\left(a^{p}+b^{p}\right)$. Hence, $(a+b)^{p} \leq 2^{p-1}\left(a^{p}+b^{p}\right)$ holds. Thus, for each $x, y, z \in X$, we obtain that

$$
\begin{aligned}
b_{d}(x, y) & =\left(d_{l}(x, y)\right)^{p} \leq\left[d_{l}(x, z)+d_{l}(z, y)\right]^{p} \\
& \leq 2^{p-1}\left[\left(d_{l}(x, z)\right)^{p}+\left(d_{l}(z, y)\right)^{p}\right] \\
& =2^{p-1}\left[b_{d}(x, z)+b_{d}(z, y)\right] .
\end{aligned}
$$

So, condition $\left(\mathrm{b}_{\mathrm{d} 3}\right)$ of Definition 1.10 is also satisfied and $b_{d}$ is a $b_{d}$-metric.

However, if $\left(X, d_{l}\right)$ is a dislocated metric space, then $\left(X, b_{d}\right)$ is not necessarily a dislocated metric space. For example, if $X=\mathbb{R}$ is the set of real numbers, then $d_{l}(x, y)=|x|+|y|$ is a dislocated metric, and $b_{d}(x, y)=(|x|+|y|)^{2}$ is a $b$-dislocated metric on $\mathbb{R}$ with $s=2$, but not a dislocated metric on $\mathbb{R}$.

Recently, Sarma and Kumari [15] established the existence of a topology induced by a dislocated metric which is metrizable with a family of sets $\{B(x, \varepsilon) \cup\{x\}: x \in X, \varepsilon>0\}$ as a base, where $B(x, \varepsilon)=\left\{y \in X: d_{l}(x, y)<\varepsilon\right\}$ for all $x \in X$ and $\varepsilon>0$. Also, $\overline{B(x, \varepsilon)}=\{y \in X$ : $\left.d_{l}(x, y) \leq \varepsilon\right\}$ is a closed ball.

On the similar lines, we show that each $b$-dislocated metric space on $X$ generates a topology $\tau_{b_{d}}$ whose base is the family of open $b_{d}$-balls

$$
B_{b_{d}}(x, \varepsilon)=\left\{y \in X: b_{d}(x, y)<\varepsilon\right\} .
$$

Definition 1.12 We say that a net $\left(x_{\alpha}: \alpha \in \Delta\right)$ in $X$ converges to $x$ in $\left(X, b_{d}\right)$ and write $\lim _{\alpha \in \Delta} x_{\alpha}=x$ if $\lim _{\alpha \in \Delta} b_{d}\left(x_{\alpha}, x\right)=0$.

Note that the limit of a net in $\left(X, b_{d}\right)$ is unique. For $A \subseteq X$, we write $D(A)=\{x \in X$ : $x$ is a limit of a net in $\left(A, b_{d}\right)$.

Proposition 1.13 If $A, B \subseteq X$, then

(i) $D(A)=\varnothing$ if $A=\varnothing$,

(ii) $D(A) \subseteq D(B)$ if $A \subseteq B$,

(iii) $D(A \cup B)=D(A) \cup D(B)$,

(iv) $D(D(A)) \subseteq D(A)$.

Proof To prove (i), (ii) and (iii), we refer to [15]. To prove (iv), let $x \in D(D(A))$. Suppose that for each $\alpha$ in $\Delta,\left(x_{\alpha_{\beta}}: \beta \in \Delta(\alpha)\right)$ is a net in $A$ such that $x_{\alpha}=\lim _{\beta \in \Delta(\alpha)} x_{\alpha_{\beta}}$. Thus, for each positive integer $i$, there is $\alpha_{i} \in \Delta$ such that $b_{d}\left(x_{\alpha_{i}}, x\right)<\frac{1}{2 i s}$, and $\beta_{i} \in \Delta\left(\alpha_{i}\right)$ such that $b_{d}\left(x_{\alpha_{i_{i}}}, x_{\alpha_{i}}\right)<\frac{1}{2 i s}$. Take $\alpha_{i_{\beta_{i}}}=\gamma_{i}$ for each $i$, then $\left\{\gamma_{1}, \gamma_{2}, \gamma_{3}, \ldots\right\}$ is a directed set $\gamma_{i}<\gamma_{j}$ if $i<j$, and $b_{d}\left(x_{\gamma_{i}}, x\right) \leq s\left(b_{d}\left(x_{\gamma_{i}}, x_{\alpha_{i}}\right)+b_{d}\left(x_{\alpha_{i}}, x\right)\right)<\frac{1}{i}$. This implies that $x \in D(A)$.

As a corollary, we have the following.

Corollary 1.14 Let, for all $A \subset X, \bar{A}=A \cup D(A)$. Then the operation $A \rightarrow \bar{A}$ on $P(X)$ satisfies Kuratowski's closure axioms [2]:

(i) $\bar{\varnothing}=\varnothing$,

(ii) $A \subset \bar{A}$,

(iii) $\bar{A}=\overline{\bar{A}}$,

(iv) $\overline{A \cup B}=\bar{A} \cup \bar{B}$. 
Consequently, we have the following.

Theorem 1.15 Let $\Upsilon$ be the family of all subsets $A$ of $X$ for which $\bar{A}=A$ and $\tau_{b_{d}}$ are the complements of members of $\Upsilon$. Then the $\tau_{b_{d}}$ is a topology for $X$ and the $\tau_{b_{d}}$-closure of a subset $A$ of $X$ is $\bar{A}$.

Definition 1.16 The topology $\tau_{b_{d}}$ obtained in Theorem 1.15 is called the topology induced by $b_{d}$ and simply referred to as the $b_{d}$-topology of $X$; and it is denoted by $\left(X, b_{d}, \tau_{b_{d}}\right)$.

Now we state some propositions and corollaries in $\left(X, b_{d}, \tau_{b_{d}}\right)$ which can be proved following similar arguments to those given in [15].

Proposition 1.17 Let $A \subseteq X$. Then $x \in D(A)$ iff for every $\delta>0, B_{\delta}(x) \cap A \neq \varnothing$.

Corollary $1.18 x \in \bar{A} \Longleftrightarrow x \in A$ or $B_{\delta}(x) \cap A \neq \varnothing, \forall \delta>0$.

Corollary 1.19 A set $A \subseteq X$ is open in $\left(X, b_{d}, \tau_{b_{d}}\right)$ if and only iffor every $x \in A$, there is $\delta>0$ such that $\{x\} \cup B_{\delta}(x) \subseteq A$.

Proposition 1.20 If $x \in X$ and $\delta>0$, then $\{x\} \cup B_{\delta}(x)$ is an open set in $\left(X, b_{d}, \tau_{b_{d}}\right)$.

Corollary 1.21 If $x \in X$ and $V_{r}(x)=B_{r}(x) \cup\{x\}$ for $r>0$, then the collection $\left\{V_{r}(x) \mid x \in X\right\}$ is an open base at $x$ in $\left(X, b_{d}, \tau_{b_{d}}\right)$. If $b_{d}$ is a $b$-metric and $V=B(x)$, then $\tau_{b_{d}}$ coincides with the metric topology.

Proposition $1.22\left(X, b_{d}, \tau_{b_{d}}\right)$ is a Hausdorff space.

Proof If $x, y \in X$ and $\frac{b_{d}(x, y)}{2 s}=r>0$, then $V_{r}(x) \cap V_{r}(y)=\varnothing$.

Corollary 1.23 If $x \in X$, then the collection $\{V(x) \mid x \in X\}$ is an open base at $x$ for $\left(X, b_{d}, \tau_{b_{d}}\right)$. Hence, $\left(X, b_{d}, \tau_{b_{d}}\right)$ is first countable.

Remark 1.24 The above corollary enables us to deal with sequences instead of nets.

Motivated by Proposition 3.2 in [11], we have the following proposition for the $b$-dislocated metric space.

Proposition 1.25 Let $\left(X, b_{d}\right)$ be a $b$-dislocated metric space. The following three conditions are equivalent:

(i) For all $x \in X$, we have $b_{d}(x, x)=0$.

(ii) $b_{d}$ is a $b$-metric.

(iii) For all $x \in X$ and all $r>0$, we have $B_{r}(x) \neq \varnothing$.

Proof We show that (iii) implies (i). Since $B \frac{r}{2 s}(x) \neq \varnothing$ for all $r>0$, there exists some $y \in X$ with $b_{d}(x, y)<\frac{r}{2 s}$. But for all $y \in X$, we have $b_{d}(x, x) \leq 2 s b_{d}(x, y)$. Therefore, $b_{d}(x, x)<r$ for all $r>0$. Hence, $b_{d}(x, x)=0$.

If $\left(X, b_{d}\right)$ is a $b$-dislocated metric space, then $\left(X^{\prime}, b_{d}\right)$, where $X^{\prime}=\left\{x \in X \mid b_{d}(x, x)=0\right\}$ is a $b$-metric space. Indeed, $\left(X^{\prime}, b_{d}\right)$ is a $b$-dislocated metric space, so assertion now follows immediately from the above proposition. 
Definition 1.26 A sequence $\left\{x_{n}\right\}$ in a $b$-dislocated metric space $\left(X, b_{d}\right)$ converges with respect to $b_{d}$ ( $b_{d}$-convergent) if there exists $x \in X$ such that $b_{d}\left(x_{n}, x\right)$ converges to 0 as $n \rightarrow \infty$. In this case, $x$ is called the limit of $\left\{x_{n}\right\}$, and we write $x_{n} \rightarrow x$.

Proposition 1.27 Limit of a convergent sequence in a b-dislocated metric space is unique.

Proof Let $x$ and $y$ be limits of the sequence $\left\{x_{n}\right\}$. By properties $\left(\mathrm{b}_{\mathrm{d} 2}\right)$ and $\left(\mathrm{b}_{\mathrm{d} 3}\right)$ of Definition 1.10, it follows that $b_{d}(x, y) \leq s\left(b_{d}\left(x_{n}, x\right)+b_{d}\left(x_{n}, y\right)\right) \rightarrow 0$. Hence, $b_{d}(x, y)=0$, and by property $\left(\mathrm{b}_{\mathrm{d} 1}\right)$ of Definition 1.10 it follows that $x=y$.

Definition 1.28 A sequence $\left\{x_{n}\right\}$ in a $b$-dislocated metric space $\left(X, b_{d}\right)$ is called a $b_{d}$ Cauchy sequence if, given $\varepsilon>0$, there exits $n_{0} \in N$ such that for all $n, m \geq n_{0}$, we have $b_{d}\left(x_{m}, x_{n}\right)<\varepsilon$ or $\lim _{n, m \rightarrow \infty} b_{d}\left(x_{n}, x_{m}\right)=0$.

Proposition 1.29 Every convergent sequence in a b-dislocated space is $b_{d}$-Cauchy.

Proof Let $\left\{x_{n}\right\}$ be a sequence which converges to some $x$, and $\varepsilon>0$. Then there exists $n_{0} \in \mathbb{N}$ with $b_{d}\left(x_{n}, x\right)<\frac{\varepsilon}{2 s}$ for all $n \geq n_{0}$. For $m, n \geq n_{0}$, we obtain $b_{d}\left(x_{n}, x_{m}\right) \leq s\left(b_{d}\left(x_{n}, x\right)+\right.$ $\left.b_{d}\left(x_{m}, x\right)\right)<2 s \frac{\varepsilon}{2 s}=\varepsilon$. Hence, $\left\{x_{n}\right\}$ is $b_{d}$-Cauchy.

Definition 1.30 A $b$-dislocated metric space $\left(X, b_{d}\right)$ is called complete if every $b_{d}$-Cauchy sequence in $X$ is $b_{d}$-convergent.

The following example shows that in general a $b$-dislocated metric is not continuous.

Example 1.31 Let $X=\mathbb{N} \cup\{\infty\}$ and $b_{d}: X \times X \rightarrow R$ be defined by

$$
b_{d}(m, n)= \begin{cases}\frac{1}{m}+\frac{1}{n} & \text { if } m, n \text { are even or } m n=\infty \\ 5 & \text { if } m \text { and } n \text { are odd and } m \neq n \\ 2 & \text { otherwise }\end{cases}
$$

Then it is easy to see that for all $m, n, p \in X$, we have

$$
b_{d}(m, p) \leq 5\left(b_{d}(m, n)+b_{d}(n, p)\right) .
$$

Thus, $\left(X, b_{d}\right)$ is a $b$-dislocated metric space. Let $x_{2 n}=2 n$ for each $n \in \mathbb{N}$. Then

$$
b_{d}(2 n, \infty)=\frac{1}{2 n} \rightarrow 0 \quad \text { as } n \rightarrow \infty,
$$

that is, $x_{n} \rightarrow \infty$, but $b_{d}\left(x_{n}, 1\right)=2 \nrightarrow b_{d}(\infty, 1)$ as $n \rightarrow \infty$.

We need the following simple lemma about the $b_{d}$-convergent sequences in the proof of our main results.

Lemma 1.32 Let $\left(X, b_{d}\right)$ be a b-dislocated metric with parameter $s \geq 1$. Suppose that $\left\{x_{n}\right\}$ and $\left\{y_{n}\right\}$ are $b_{d}$-convergent to $x, y$, respectively. Then we have

$$
\frac{1}{s^{2}} b_{d}(x, y) \leq \liminf _{n \rightarrow \infty} b_{d}\left(x_{n}, y_{n}\right) \leq \limsup _{n \rightarrow \infty} b_{d}\left(x_{n}, y_{n}\right) \leq s^{2} b_{d}(x, y) .
$$


In particular, if $b_{d}(x, y)=0$, then we have $\lim _{n \rightarrow \infty} b_{d}\left(x_{n}, y_{n}\right)=0=b_{d}(x, y)$. Moreover, for each $z \in X$, we have

$$
\frac{1}{s} b_{d}(x, z) \leq \liminf _{n \rightarrow \infty} b_{d}\left(x_{n}, z\right) \leq \limsup _{n \rightarrow \infty} b_{d}\left(x_{n}, z\right) \leq s b_{d}(x, z) .
$$

In particular, if $b_{d}(x, z)=0$, then we have $\lim _{n \rightarrow \infty} b_{d}\left(x_{n}, z\right)=0=b_{d}(x, z)$.

Proof Using the triangle inequality in a $b$-dislocated metric space, it is easy to see that

$$
b_{d}(x, y) \leq s b_{d}\left(x, x_{n}\right)+s^{2} b_{d}\left(x_{n}, y_{n}\right)+s^{2} b_{d}\left(y_{n}, y\right)
$$

and

$$
b_{d}\left(x_{n}, y_{n}\right) \leq s b_{d}\left(x_{n}, x\right)+s^{2} b_{d}(x, y)+s^{2} b_{d}\left(y, y_{n}\right) .
$$

Taking the lower limit as $n \rightarrow \infty$ in the first inequality and the upper limit as $n \rightarrow \infty$ in the second inequality, the result follows. Similarly, using again the triangle inequality, the last assertion follows.

Definition 1.33 [3] Let $f$ and $g$ be two self-maps on a nonempty set $X$. If $w=f x=g x$, for some $x$ in $X$, then $x$ is called a coincidence point of $f$ and $g$, where $w$ is called a point of coincidence of $f$ and $g$.

Definition 1.34 [3] Let $f$ and $g$ be two self-maps defined on a set $X$. Then $f$ and $g$ are said to be weakly compatible if they commute at every coincidence point.

Definition 1.35 Let $\left(X, b_{d}\right)$ be a $b$-dislocated metric space. Then the pair $(f, g)$ is said to be compatible if and only if $\lim _{n \rightarrow \infty} b_{d}\left(f g x_{n}, g f x_{n}\right)=0$, whenever $\left\{x_{n}\right\}$ is a sequence in $X$ so that $\lim _{n \rightarrow \infty} f x_{n}=\lim _{n \rightarrow \infty} g x_{n}=t$ for some $t \in X$.

\section{Common fixed point results}

Suppose that

$$
\begin{aligned}
\Psi= & \{\psi:[0, \infty) \rightarrow[0, \infty) \mid \psi \text { is a continuous non-decreasing function with } \\
& \psi(t)=0 \Leftrightarrow t=0\}
\end{aligned}
$$

and

$$
\begin{gathered}
\Phi=\{\varphi:[0, \infty) \rightarrow[0, \infty) \mid \varphi \text { is a lower semi-continuous function with } \\
\varphi(t)=0 \Leftrightarrow t=0\} .
\end{gathered}
$$

Theorem 2.1 Let $\left(X, b_{d}, \preceq\right)$ be an ordered complete b-dislocated metric space, and let $f$, $g, S$ and $T$ be four self-maps on $X$ such that $(f, g)$ and $(S, T)$ are dominated and dominating maps, respectively, with $f X \subseteq T X$ and $g X \subseteq S X$. Suppose that for all two comparable elements $x, y \in X$,

$$
\psi\left(2 s^{4} b_{d}(f x, g y)\right) \leq \psi\left(M_{s}(x, y)\right)-\varphi\left(M_{s}(x, y)\right)
$$


is satisfied, where

$$
M_{s}(x, y)=\max \left\{b_{d}(S x, T y), b_{d}(f x, S x), b_{d}(g y, T y), \frac{b_{d}(S x, g y)+b_{d}(f x, T y)}{4 s}\right\},
$$

$\psi \in \Psi$ and $\varphi \in \Phi$.Iffor every non-increasing sequence $\left\{x_{n}\right\}$ and a sequence $\left\{y_{n}\right\}$ with $y_{n} \preceq$ $x_{n}$, for all $n$ such that $y_{n} \rightarrow u$, we have $u \preceq x_{n}$ and either

$\left(\mathrm{a}_{1}\right)(f, S)$ are compatible, $f$ or $S$ is continuous and $(g, T)$ is weakly compatible, or $\left(\mathrm{a}_{2}\right)(g, T)$ are compatible, $g$ or $T$ is continuous and $(f, S)$ is weakly compatible,

then $f, g, S$ and $T$ have a common fixed point. Moreover, the set of common fixed points of $f, g, S$ and $T$ is well ordered if and only iff, $g, S$ and $T$ have one and only one common fixed point.

Proof Let $x_{0}$ be an arbitrary point in $X$. We define inductively the sequences $\left\{x_{n}\right\}$ and $\left\{y_{n}\right\}$ in $X$ by

$$
y_{2 n+1}=f x_{2 n}=T x_{2 n+1}, \quad y_{2 n+2}=g x_{2 n+1}=S x_{2 n+2}, \quad n=0,1,2, \ldots
$$

This can be done as $f X \subseteq T X$ and $g X \subseteq S X$. By given assumptions, $x_{2 n+1} \preceq T x_{2 n+1}=f x_{2 n} \preceq$ $x_{2 n}$ and $x_{2 n} \preceq S x_{2 n}=g x_{2 n-1} \preceq x_{2 n-1}$. Thus, we have $x_{n+1} \preceq x_{n}$ for all $n \geq 0$. We will show that $\left\{y_{n}\right\}$ is $b_{d}$-Cauchy. Suppose that $b_{d}\left(y_{2 n}, y_{2 n+1}\right)>0$ for every $n$. If not, then for some $k$, $b_{d}\left(y_{2 k}, y_{2 k+1}\right)=0$, and from (2.1), we obtain

$$
\begin{aligned}
\psi\left(b_{d}\left(y_{2 k+1}, y_{2 k+2}\right)\right) & \leq \psi\left(2 s^{4} b_{d}\left(y_{2 k+1}, y_{2 k+2}\right)\right) \\
& =\psi\left(2 s^{4} b_{d}\left(x_{2 k}, g x_{2 k+1}\right)\right) \\
& \leq \psi\left(M_{s}\left(x_{2 k}, x_{2 k+1}\right)\right)-\varphi\left(M_{s}\left(x_{2 k}, x_{2 k+1}\right)\right)
\end{aligned}
$$

where

$$
\begin{aligned}
M_{s}\left(x_{2 k}, x_{2 k+1}\right)= & \max \left\{b_{d}\left(S x_{2 k}, T x_{2 k+1}\right), b_{d}\left(f x_{2 k}, S x_{2 k}\right), b_{d}\left(g x_{2 k+1}, T x_{2 k+1}\right),\right. \\
& \left.\frac{b_{d}\left(S x_{2 k}, g x_{2 k+1}\right)+b_{d}\left(f x_{2 k}, T x_{2 k+1}\right)}{4 s}\right\} \\
= & \max \left\{b_{d}\left(y_{2 k}, y_{2 k+1}\right), b_{d}\left(y_{2 k+1}, y_{2 k}\right), b_{d}\left(y_{2 k+2}, y_{2 k+1}\right),\right. \\
& \left.\frac{b_{d}\left(y_{2 k}, y_{2 k+2}\right)+b_{d}\left(y_{2 k+1}, y_{2 k+1}\right)}{4 s}\right\} \\
= & \max \left\{0,0, b_{d}\left(y_{2 k+1}, y_{2 k+2}\right), \frac{b_{d}\left(y_{2 k}, y_{2 k+2}\right)+b_{d}\left(y_{2 k+1}, y_{2 k+1}\right)}{4 s}\right\} \\
= & b_{d}\left(y_{2 k+1}, y_{2 k+2}\right),
\end{aligned}
$$

since

$$
\begin{aligned}
\frac{b_{d}\left(y_{2 k}, y_{2 k+2}\right)+b_{d}\left(y_{2 k+1}, y_{2 k+1}\right)}{4 s} & \leq \frac{s b_{d}\left(y_{2 k}, y_{2 k+1}\right)+s b_{d}\left(y_{2 k+1}, y_{2 k+2}\right)+2 s b_{d}\left(y_{2 k}, y_{2 k+1}\right)}{4 s} \\
& =\frac{s b_{d}\left(y_{2 k+1}, y_{2 k+2}\right)}{4 s}<b_{d}\left(y_{2 k+1}, y_{2 k+2}\right) .
\end{aligned}
$$


So, from (2.3) and (2.4), we obtain that

$$
\psi\left(b_{d}\left(y_{2 k+1}, y_{2 k+2}\right)\right) \leq \psi\left(b_{d}\left(y_{2 k+1}, y_{2 k+2}\right)\right)-\varphi\left(b_{d}\left(y_{2 k+1}, y_{2 k+2}\right)\right),
$$

which gives $\varphi\left(b_{d}\left(y_{2 k+1}, y_{2 k+2}\right)\right) \leq 0$ and so $y_{2 k+1}=y_{2 k+2}$, which further implies that $y_{2 k+2}=$ $y_{2 k+3}$. Thus, $\left\{y_{n}\right\}$ becomes a constant sequence, hence, $y_{n}$ is a Cauchy sequence.

Now, take $b_{d}\left(y_{2 n}, y_{2 n+1}\right)>0$ for each $n$. As $x_{2 n}$ and $x_{2 n+1}$ are comparable, so from (2.1) we have

$$
\begin{aligned}
\psi\left(b_{d}\left(y_{2 n+1}, y_{2 n+2}\right)\right) & \leq \psi\left(2 s^{4} b_{d}\left(y_{2 n+1}, y_{2 n+2}\right)\right) \\
& =\psi\left(2 s^{4} b_{d}\left(f x_{2 n}, g x_{2 n+1}\right)\right) \\
& \leq \psi\left(M_{s}\left(x_{2 n}, x_{2 n+1}\right)\right)-\varphi\left(M_{s}\left(x_{2 n}, x_{2 n+1}\right)\right) \\
& \leq \psi\left(M_{s}\left(x_{2 n}, x_{2 n+1}\right)\right)
\end{aligned}
$$

Hence

$$
b_{d}\left(y_{2 n+1}, y_{2 n+2}\right) \leq M_{s}\left(x_{2 n}, x_{2 n+1}\right)
$$

where

$$
\begin{aligned}
M_{s}\left(x_{2 n}, x_{2 n+1}\right)= & \max \left\{b_{d}\left(S x_{2 n}, T x_{2 n+1}\right), b_{d}\left(f x_{2 n}, S x_{2 n}\right), b_{d}\left(g x_{2 n+1}, T x_{2 n+1}\right),\right. \\
& \left.\frac{b_{d}\left(S x_{2 n}, g x_{2 n+1}\right)+b_{d}\left(f x_{2 n}, T x_{2 n+1}\right)}{4 s}\right\} \\
= & \max \left\{b_{d}\left(y_{2 n}, y_{2 n+1}\right), b_{d}\left(y_{2 n+1}, y_{2 n}\right), b_{d}\left(y_{2 n+2}, y_{2 n+1}\right),\right. \\
& \left.\frac{b_{d}\left(y_{2 n}, y_{2 n+2}\right)+b_{d}\left(y_{2 n+1}, y_{2 n+1}\right)}{4 s}\right\} \\
\leq & \max \left\{b_{d}\left(y_{2 n}, y_{2 n+1}\right), b_{d}\left(y_{2 n+1}, y_{2 n+2}\right),\right. \\
& \left.\frac{\left.s b_{d}\left(y_{2 n}, y_{2 n+1}\right)+s b_{d}\left(y_{2 n+1}, y_{2 n+2}\right)+2 s b_{d}\left(y_{2 n}, y_{2 n+1}\right)\right\}}{4 s}\right\} \\
= & \max \left\{b_{d}\left(y_{2 n}, y_{2 n+1}\right), b_{d}\left(y_{2 n+1}, y_{2 n+2}\right),\right. \\
& \left.\frac{\left.3 s b_{d}\left(y_{2 n}, y_{2 n+1}\right)+s b_{d}\left(y_{2 n+1}, y_{2 n+2}\right)\right\}}{4 s}\right\} \\
= & \max \left\{b_{d}\left(y_{2 n}, y_{2 n+1}\right), b_{d}\left(y_{2 n+1}, y_{2 n+2}\right)\right\} .
\end{aligned}
$$

If for some $n, b_{d}\left(y_{2 n+1}, y_{2 n+2}\right) \geq b_{d}\left(y_{2 n}, y_{2 n+1}\right)>0$, then (2.6) gives that $M_{s}\left(x_{2 n}, x_{2 n+1}\right)=$ $b_{d}\left(y_{2 n+1}, y_{2 n+2}\right)$ and from (2.1) we have

$$
\begin{aligned}
\psi\left(b_{d}\left(y_{2 n+1}, y_{2 n+2}\right)\right) & \leq \psi\left(2 s^{4} b_{d}\left(y_{2 n+1}, y_{2 n+2}\right)\right) \\
& \leq \psi\left(M_{s}\left(x_{2 n}, x_{2 n+1}\right)\right)-\varphi\left(M_{s}\left(x_{2 n}, x_{2 n+1}\right)\right) \\
& =\psi\left(b_{d}\left(y_{2 n+1}, y_{2 n+2}\right)\right)-\varphi\left(b_{d}\left(y_{2 n+1}, y_{2 n+2}\right)\right)
\end{aligned}
$$


which yields that $\varphi\left(b_{d}\left(y_{2 n+1}, y_{2 n+2}\right)\right) \leq 0$, or, equivalently, $b_{d}\left(y_{2 n+1}, y_{2 n+2}\right)=0$, a contradiction.

Hence, $M_{s}\left(x_{2 n}, x_{2 n+1}\right) \leq b_{d}\left(y_{2 n}, y_{2 n+1}\right)$. Since $M_{s}\left(x_{2 n}, x_{2 n+1}\right) \geq b_{d}\left(y_{2 n}, y_{2 n+1}\right)$, therefore, $b_{d}\left(y_{2 n+1}, y_{2 n+2}\right) \leq M_{s}\left(x_{2 n}, x_{2 n+1}\right)=b_{d}\left(y_{2 n}, y_{2 n+1}\right)$. Following similar arguments to those given above, we have

$$
b_{d}\left(y_{2 n+2}, y_{2 n+3}\right) \leq M_{s}\left(x_{2 n+1}, x_{2 n+2}\right)=b_{d}\left(y_{2 n+1}, y_{2 n+2}\right) .
$$

Therefore, $\left\{b_{d}\left(y_{n}, y_{n+1}\right)\right\}$ is a non-increasing sequence and so there exists $r \geq 0$ such that

$$
\lim _{n \rightarrow \infty} b_{d}\left(y_{n-1}, y_{n}\right)=\lim _{n \rightarrow \infty} M_{s}\left(x_{n}, x_{n+1}\right)=r .
$$

Suppose that $r>0$. As

$$
\begin{aligned}
\psi\left(b_{d}\left(y_{2 n+1}, y_{2 n+2}\right)\right) & \leq \psi\left(2 s^{4} b_{d}\left(y_{2 n+1}, y_{2 n+2}\right)\right) \\
& \leq \psi\left(M_{s}\left(x_{2 n}, x_{2 n+1}\right)\right)-\varphi\left(M_{s}\left(x_{2 n}, x_{2 n+1}\right)\right)
\end{aligned}
$$

by taking the upper limit as $n \rightarrow \infty$, we obtain

$$
\begin{aligned}
\psi(r) & \leq \psi(r)-\liminf _{n \rightarrow \infty} \varphi\left(M_{s}\left(x_{2 n}, x_{2 n+1}\right)\right) \\
& =\psi(r)-\varphi\left(\liminf _{n \rightarrow \infty} M_{s}\left(x_{2 n}, x_{2 n+1}\right)\right) \\
& =\psi(r)-\varphi(r),
\end{aligned}
$$

a contradiction. Hence

$$
\lim _{n \rightarrow \infty} b_{d}\left(y_{n-1}, y_{n}\right)=0 .
$$

Now, we prove that $\left\{y_{n}\right\}$ is a $b_{d}$-Cauchy sequence. To do this, it is sufficient to show that the subsequence $\left\{y_{2 n}\right\}$ is $b_{d}$-Cauchy in $X$. Assume on the contrary that $\left\{y_{2 n}\right\}$ is not a $b_{d}$-Cauchy sequence. Then there exists $\varepsilon>0$ for which we can find subsequences $\left\{y_{2 m_{k}}\right\}$ and $\left\{y_{2 n_{k}}\right\}$ of $\left\{y_{2 n}\right\}$ so that $n_{k}$ is the smallest index for which $2 n_{k}>2 m_{k}>k$,

$$
b_{d}\left(y_{2 m_{k}}, y_{2 n_{k}}\right) \geq \varepsilon
$$

and

$$
b_{d}\left(y_{2 m_{k}}, y_{2 n_{k}-2}\right)<\varepsilon
$$

Using the triangle inequality and (2.10), we obtain that

$$
\varepsilon \leq b_{d}\left(y_{2 m_{k}}, y_{2 n_{k}}\right) \leq s b_{d}\left(y_{2 m_{k}}, y_{2 m_{k}+1}\right)+s b_{d}\left(y_{2 m_{k}+1}, y_{2 n_{k}}\right) .
$$

Taking the upper limit as $k \rightarrow \infty$ and using (2.8), we obtain

$$
\frac{\varepsilon}{s} \leq \limsup _{k \rightarrow \infty} b_{d}\left(y_{2 m_{k}+1}, y_{2 n_{k}}\right) \text {. }
$$


Using the triangle inequality and (2.10), we have

$$
\begin{aligned}
\varepsilon & \leq b_{d}\left(y_{2 m_{k}}, y_{2 n_{k}}\right) \\
& \leq s b_{d}\left(y_{2 m_{k}}, y_{2 n_{k}-2}\right)+s^{2} b_{d}\left(y_{2 n_{k}-2}, y_{2 n_{k}-1}\right)+s^{2} b_{d}\left(y_{2 n_{k}-1}, y_{2 n_{k}}\right) \\
& <\varepsilon s+s^{2} b_{d}\left(y_{2 n_{k}-2}, y_{2 n_{k}-1}\right)+s^{2} b_{d}\left(y_{2 n_{k}-1}, y_{2 n_{k}}\right) .
\end{aligned}
$$

Taking the upper limit as $k \rightarrow \infty$ and using (2.8), we obtain

$$
\varepsilon \leq \limsup _{k \rightarrow \infty} b_{d}\left(y_{2 m_{k}}, y_{2 n_{k}}\right) \leq \varepsilon s
$$

Also,

$$
\varepsilon \leq b_{d}\left(y_{2 m_{k}}, y_{2 n_{k}}\right) \leq s b_{d}\left(y_{2 m_{k}}, y_{2 n_{k}-1}\right)+s b_{d}\left(y_{2 n_{k}-1}, y_{2 n_{k}}\right) .
$$

Hence

$$
\frac{\varepsilon}{s} \leq \limsup _{k \rightarrow \infty} b_{d}\left(y_{2 m_{k}}, y_{2 n_{k}-1}\right)
$$

On the other hand, we have

$$
b_{d}\left(y_{2 m_{k}}, y_{2 n_{k}-1}\right) \leq s b_{d}\left(y_{2 m_{k}}, y_{2 n_{k}}\right)+s b_{d}\left(y_{2 n_{k}}, y_{2 n_{k}-1}\right) .
$$

So, from (2.8) and (2.12), we have

$$
\limsup _{k \rightarrow \infty} b_{d}\left(y_{2 m_{k}}, y_{2 n_{k}-1}\right) \leq s \limsup _{k \rightarrow \infty} b_{d}\left(y_{2 m_{k}}, y_{2 n_{k}}\right) \leq \varepsilon s^{2} .
$$

Consequently,

$$
\frac{\varepsilon}{s} \leq \limsup _{k \rightarrow \infty} b_{d}\left(y_{2 m_{k}}, y_{2 n_{k}-1}\right) \leq \varepsilon s^{2} .
$$

Similarly,

$$
\frac{\varepsilon}{s^{2}} \leq \limsup _{k \rightarrow \infty} b_{d}\left(y_{2 m_{k}+1}, y_{2 n_{k}-1}\right) \leq \varepsilon s^{2} .
$$

As $x_{2 m_{k}}$ and $x_{2 n_{k}-1}$ are comparable, from (2.1) we have

$$
\begin{aligned}
\psi\left(2 s^{4} b_{d}\left(y_{2 m_{k}+1}, y_{2 n_{k}}\right)\right) & =\psi\left(2 s^{4} b_{d}\left(f x_{2 m_{k}}, g x_{2 n_{k}-1}\right)\right) \\
& \leq \psi\left(M_{s}\left(x_{2 m_{k}}, x_{2 n_{k}-1}\right)\right)-\varphi\left(M_{s}\left(x_{2 m_{k}}, x_{2 n_{k}-1}\right)\right),
\end{aligned}
$$

where

$$
\begin{aligned}
M_{s}\left(x_{2 m_{k}}, x_{2 n_{k}-1}\right)= & \max \left\{b_{d}\left(S x_{2 m_{k}}, T x_{2 n_{k}-1}\right), b_{d}\left(f x_{2 m_{k}}, S x_{2 m_{k}}\right), b_{d}\left(g x_{2 n_{k}-1}, T x_{2 n_{k}-1}\right),\right. \\
& \left.\frac{b_{d}\left(S x_{2 m_{k}}, g x_{2 n_{k}-1}\right)+b_{d}\left(f x_{2 m_{k}}, T x_{2 n_{k}-1}\right)}{4 s}\right\}
\end{aligned}
$$




$$
\begin{aligned}
= & \max \left\{b_{d}\left(y_{2 m_{k}}, y_{2 n_{k}-1}\right), b_{d}\left(y_{2 m_{k}+1}, y_{2 m_{k}}\right), b_{d}\left(y_{2 n_{k}}, y_{2 n_{k}-1}\right),\right. \\
& \left.\frac{b_{d}\left(y_{2 m_{k}}, y_{2 n_{k}}\right)+b_{d}\left(y_{2 m_{k}+1}, y_{2 n_{k}-1}\right)}{4 s}\right\} .
\end{aligned}
$$

Taking the upper limit and using (2.8) and (2.12)-(2.13), we get

$$
\begin{aligned}
\frac{\varepsilon+\frac{\varepsilon}{s^{2}}}{4 s}= & \min \left\{\frac{\varepsilon}{s}, \frac{\varepsilon+\frac{\varepsilon}{s^{2}}}{4 s}\right\} \\
\leq & \limsup _{k \rightarrow \infty} M_{s}\left(x_{2 m_{k}}, x_{2 n_{k}-1}\right) \\
= & \max \left\{\limsup _{k \rightarrow \infty} b_{d}\left(y_{2 m_{k}}, y_{2 n_{k}-1}\right), 0,0,\right. \\
& \left.\frac{\limsup _{k \rightarrow \infty} b_{d}\left(y_{2 m_{k}}, y_{2 n_{k}}\right)+\limsup _{k \rightarrow \infty} b_{d}\left(y_{2 m_{k}+1}, y_{2 n_{k}-1}\right)}{4 s}\right\} \\
\leq & \max \left\{\varepsilon s^{2}, \frac{\varepsilon s+\varepsilon s^{2}}{4 s}\right\}=\varepsilon s^{2} .
\end{aligned}
$$

Hence, we have

$$
\frac{\varepsilon+\frac{\varepsilon}{s^{2}}}{4 s} \leq \limsup _{k \rightarrow \infty} M_{s}\left(x_{2 m_{k}}, x_{2 n_{k}-1}\right) \leq \varepsilon s^{2} .
$$

Similarly, we can obtain

$$
\frac{\varepsilon+\frac{\varepsilon}{s^{2}}}{4 s} \leq \liminf _{k \rightarrow \infty} M_{s}\left(x_{2 m_{k}}, x_{2 n_{k}-1}\right) \leq \varepsilon s^{2} .
$$

As

$$
\begin{aligned}
\psi\left(2 s^{4} b_{d}\left(y_{2 m_{k}+1}, y_{2 n_{k}}\right)\right) & =\psi\left(2 s^{4} b_{d}\left(f x_{2 m_{k}}, g x_{2 n_{k}-1}\right)\right) \\
& \leq \psi\left(M_{s}\left(x_{2 m_{k}}, x_{2 n_{k}-1}\right)\right)-\varphi\left(M_{s}\left(x_{2 m_{k}}, x_{2 n_{k}-1}\right)\right),
\end{aligned}
$$

so, by taking the upper limit as $k \rightarrow \infty$, and from (2.11) and (2.15), we obtain

$$
\begin{aligned}
\psi\left(2 \varepsilon s^{3}\right) & =\psi\left(2 s^{4} \frac{\varepsilon}{s}\right) \\
& \leq \psi\left(2 s^{4} \limsup _{k \rightarrow \infty} b_{d}\left(y_{2 m_{k}+1}, y_{2 n_{k}}\right)\right) \\
& \leq \psi\left(\limsup _{k \rightarrow \infty} M_{s}\left(x_{2 m_{k}}, x_{2 n_{k}-1}\right)\right)-\liminf _{k \rightarrow \infty} \varphi\left(M_{s}\left(x_{2 m_{k}}, x_{2 n_{k}-1}\right)\right) \\
& \leq \psi\left(\varepsilon s^{2}\right)-\varphi\left(\liminf _{k \rightarrow \infty} M_{s}\left(x_{2 m_{k}}, x_{2 n_{k}-1}\right)\right) \\
& \leq \psi\left(2 \varepsilon s^{3}\right)-\varphi\left(\liminf _{k \rightarrow \infty} M_{s}\left(x_{2 m_{k}}, x_{2 n_{k}-1}\right)\right),
\end{aligned}
$$

which implies that

$$
\varphi\left(\liminf _{k \rightarrow \infty} M_{s}\left(x_{2 m_{k}}, x_{2 n_{k}-1}\right)\right)=0,
$$


so $\liminf M_{s}\left(x_{2 m_{k}}, x_{2 n_{k}-1}\right)=0$, a contradiction to (2.16). Hence $\left\{y_{2 n}\right\}$ is a $b_{d}$-Cauchy sequence in $X$. Since $X$ is complete, there exists $y \in X$ such that

$$
\lim _{n \rightarrow \infty} f x_{2 n}=\lim _{n \rightarrow \infty} T x_{2 n+1}=\lim _{n \rightarrow \infty} g x_{2 n+1}=\lim _{n \rightarrow \infty} S x_{2 n}=y .
$$

Now, we show that $y$ is a common fixed point of $f, g, S$ and $T$.

Assume that $\left(\mathrm{a}_{1}\right)$ holds and $S$ is continuous. Then

$$
\lim _{n \rightarrow \infty} S^{2} x_{2 n+2}=S y \quad \text { and } \quad \lim _{n \rightarrow \infty} S f x_{2 n}=S y .
$$

Using the triangle inequality, we have

$$
b_{d}\left(f S x_{2 n}, S y\right) \leq s\left(b_{d}\left(f S x_{2 n}, S f x_{2 n}\right)+b_{d}\left(S f x_{2 n}, S y\right)\right) .
$$

Since the pair $(f, S)$ is compatible, $\lim _{n \rightarrow \infty} b_{d}\left(f S x_{2 n}, S f x_{2 n}\right)=0$. So, by taking the limit when $n \rightarrow \infty$ in the above inequality, we have

$$
\lim _{n \rightarrow \infty} b_{d}\left(f S x_{2 n}, S y\right) \leq s\left(\lim _{n \rightarrow \infty} b_{d}\left(f S x_{2 n}, S f x_{2 n}\right)+\lim _{n \rightarrow \infty} b_{d}\left(S f x_{2 n}, S y\right)\right)=0 .
$$

Hence, $\lim _{n \rightarrow \infty} f S x_{2 n}=S y$. As $S x_{2 n+2}=g x_{2 n+1} \preceq x_{2 n+1}$, from (2.1) we obtain

$$
\psi\left(2 s^{4} b_{d}\left(f S x_{2 n+2}, g x_{2 n+1}\right)\right) \leq \psi\left(M_{s}\left(S x_{2 n+2}, x_{2 n+1}\right)\right)-\varphi\left(M_{s}\left(S x_{2 n+2}, x_{2 n+1}\right)\right),
$$

where

$$
\begin{aligned}
M_{s}\left(S x_{2 n+2}, x_{2 n+1}\right) & \\
= & \max \left\{b_{d}\left(S^{2} x_{2 n+2}, T x_{2 n+1}\right), b_{d}\left(f S x_{2 n+2}, S^{2} x_{2 n+2}\right), b_{d}\left(g x_{2 n+1}, T x_{2 n+1}\right),\right. \\
& \left.\frac{b_{d}\left(S^{2} x_{2 n+2}, g x_{2 n+1}\right)+b_{d}\left(f S x_{2 n+2}, T x_{2 n+1}\right)}{4 s}\right\} .
\end{aligned}
$$

Now, by using Lemma 1.32, we get

$$
\begin{aligned}
\limsup _{n \rightarrow \infty} M_{s}\left(S x_{2 n+2}, x_{2 n+1}\right) & \leq \max \left\{s^{2} b_{d}(S y, y), 0,0, \frac{s^{2} b_{d}(S y, y)+s^{2} b_{d}(S y, y)}{4 s}\right\} \\
& =s^{2} b_{d}(S y, y) .
\end{aligned}
$$

Hence, by taking the upper limit in (2.17) and using Lemma 1.32, we obtain

$$
\begin{aligned}
\psi\left(2 s^{2} b_{d}(S y, y)\right) & =\psi\left(2 s^{4} \frac{1}{s^{2}} b_{d}(S y, y)\right) \leq \psi\left(s^{2} b_{d}(S y, y)\right)-\varphi\left(s^{2} b_{d}(S y, y)\right) \\
& \leq \psi\left(2 s^{2} b_{d}(S y, y)\right)-\varphi\left(s^{2} b_{d}(S y, y)\right)
\end{aligned}
$$

which gives $\varphi\left(s^{2} b_{d}(S y, y)\right) \leq 0$, or, equivalently, $S y=y$. 
Now, since $g x_{2 n+1} \preceq x_{2 n+1}$ and $g x_{2 n+1} \rightarrow y$ as $n \rightarrow \infty$, then $y \preceq x_{2 n+1}$ and from (2.1) we have

$$
\psi\left(2 s^{4} b_{d}\left(f y, g x_{2 n+1}\right)\right) \leq \psi\left(M_{s}\left(y, x_{2 n+1}\right)\right)-\varphi\left(M_{s}\left(y, x_{2 n+1}\right)\right)
$$

where

$$
\begin{aligned}
M_{s}\left(y, x_{2 n+1}\right)= & \max \left\{b_{d}\left(S y, T x_{2 n+1}\right), b_{d}(f y, S y), b_{d}\left(g x_{2 n+1}, T x_{2 n+1}\right),\right. \\
& \left.\frac{b_{d}\left(S y, g x_{2 n+1}\right)+b_{d}\left(f y, T x_{2 n+1}\right)}{4 s}\right\} .
\end{aligned}
$$

Taking the upper limit as $n \rightarrow \infty$ in (2.18) and using Lemma 1.32, we have

$$
\begin{aligned}
\psi\left(2 s^{3} b_{d}(f y, y)\right) & =\psi\left(2 s^{4} \frac{1}{s} b_{d}(f y, y)\right) \leq \psi\left(b_{d}(f y, y)\right)-\varphi\left(b_{d}(f y, y)\right) \\
& \leq \psi\left(2 s^{3} b_{d}(f y, y)\right)-\varphi\left(b_{d}(f y, y)\right)
\end{aligned}
$$

which implies that $\varphi\left(b_{d}(f y, y)\right) \leq 0$, so $f y=y$.

Since $f(X) \subseteq T(X)$, there exists a point $v \in X$ such that $f y=T v$. Suppose that $g v \neq T v$. Since $v \preceq T v=f y \preceq y$, from $(2.1)$ we have

$$
\psi\left(b_{d}(T v, g v)\right)=\psi\left(b_{d}(f y, g v)\right) \leq \psi\left(M_{s}(y, v)\right)-\varphi\left(M_{s}(y, v)\right)
$$

where

$$
\begin{aligned}
M_{s}(y, v) & =\max \left\{b_{d}(S y, T v), b_{d}(f y, S y), b_{d}(g v, T v), \frac{b_{d}(S y, g v)+b_{d}(f y, T v)}{4 s}\right\} \\
& =b_{d}(g v, T v) .
\end{aligned}
$$

So, from (2.19) we have

$$
\psi\left(b_{d}(T v, g v)\right) \leq \psi\left(b_{d}(g v, T v)\right)-\varphi\left(b_{d}(g v, T v)\right)
$$

a contradiction. Therefore $g v=T v$. Since the pair $(g, T)$ is weakly compatible, $g y=g f y=$ $g T v=T g v=T f y=T y$ and $y$ is the coincidence point of $g$ and $T$. Since $S x_{2 n} \preceq x_{2 n}$ and $S x_{2 n} \rightarrow$ $y$ as $n \rightarrow \infty$, it implies that $y \preceq x_{2 n}$ and from (2.1) we obtain

$$
\psi\left(2 s^{4} b_{d}\left(f x_{2 n}, g y\right)\right) \leq \psi\left(M_{s}\left(x_{2 n}, y\right)\right)-\varphi\left(M_{s}\left(x_{2 n}, y\right)\right)
$$

where

$$
\begin{aligned}
M_{s}\left(x_{2 n}, y\right)= & \max \left\{b_{d}\left(S x_{2 n}, T y\right), b_{d}\left(f x_{2 n}, S x_{2 n}\right), b_{d}(g y, T y),\right. \\
& \left.\frac{b_{d}\left(S x_{2 n}, g y\right)+b_{d}\left(f x_{2 n}, T y\right)}{4 s}\right\} .
\end{aligned}
$$


Taking the upper limit as $n \rightarrow \infty$ in (2.21) and using Lemma 1.32, we have

$$
\begin{aligned}
& \max \left\{\frac{1}{s} b_{d}(y, g y), b_{d}(g y, T y), \frac{2 s}{4 s} b_{d}(y, g y)\right\} \\
& \leq \liminf _{n \rightarrow \infty} M_{s}\left(x_{2 n}, y\right) \\
& \quad \leq \limsup _{n \rightarrow \infty} M_{s}\left(x_{2 n}, y\right) \\
& \quad \leq \max \left\{s b_{d}(y, g y), b_{d}(g y, T y), \frac{2 s}{4 s} b_{d}(y, g y)\right\} \\
& \quad=\max \left\{s b_{d}(y, g y), b_{d}(g y, g y)\right\} \\
& \quad \leq \max \left\{s b_{d}(y, g y), 2 s b_{d}(y, g y)\right\} \\
& \quad=2 s b_{d}(y, g y) .
\end{aligned}
$$

Taking the upper limit as $n \rightarrow \infty$ in (2.20) and using Lemma 1.32 and (2.22), we have

$$
\begin{aligned}
\psi\left(2 s^{3} b_{d}(y, g y)\right) & =\psi\left(2 s^{4} \frac{1}{s} b_{d}(y, g y)\right) \\
& \leq \psi\left(\limsup _{n \rightarrow \infty} M_{s}\left(x_{2 n}, y\right)\right)-\liminf _{n \rightarrow \infty} \varphi\left(M_{s}\left(x_{2 n}, y\right)\right) \\
& \leq \psi\left(2 s b_{d}(y, g y)\right)-\varphi\left(\liminf _{n \rightarrow \infty} M_{s}\left(x_{2 n}, y\right)\right) \\
& \leq \psi\left(2 s^{3} b_{d}(y, g y)\right)-\varphi\left(\liminf _{n \rightarrow \infty} M_{s}\left(x_{2 n}, y\right)\right)
\end{aligned}
$$

which implies that $\liminf _{n \rightarrow \infty} M_{s}\left(x_{2 n}, y\right)=0$, so we have $y=g y$. Therefore, $f y=g y=S y=$ $T y=y$.

The proof is similar when $f$ is continuous.

Similarly, if $\left(\mathrm{a}_{2}\right)$ holds, then the result follows.

Now, suppose that the set of common fixed points of $f, g, S$ and $T$ is well ordered. We show that they have a unique common fixed point. Assume on the contrary that $f u=g u=$ $S u=T u=u$ and $f v=g v=S v=T v=v$, but $u \neq v$. By assumption, we can apply (2.1) to obtain

$$
\begin{aligned}
\psi\left(2 s b_{d}(u, v)\right) & =\psi\left(2 s b_{d}(f u, g v)\right) \\
& \leq \psi\left(2 s^{4} b_{d}(f u, g v)\right) \leq \psi\left(M_{s}(u, v)\right)-\varphi\left(M_{s}(u, v)\right),
\end{aligned}
$$

where

$$
\begin{aligned}
M_{s}(u, v) & =\max \left\{b_{d}(S u, T v), b_{d}(f u, S u), b_{d}(g v, T v), \frac{b_{d}(S u, g v)+b_{d}(f u, T v)}{4 s}\right\} \\
& =\max \left\{b_{d}(u, v), b_{d}(u, u), b_{d}(v, v), \frac{b_{d}(u, v)+b_{d}(u, v)}{4 s}\right\} \\
& =\max \left\{b_{d}(u, v), b_{d}(u, u), b_{d}(v, v)\right\} \\
& \leq \max \left\{b_{d}(u, v), 2 s b_{d}(u, v), 2 s b_{d}(u, v)\right\} \\
& \leq 2 s b_{d}(u, v) .
\end{aligned}
$$


Hence

$$
\psi\left(2 s b_{d}(u, v)\right) \leq \psi\left(2 s b_{d}(u, v)\right)-\varphi\left(M_{s}(u, v)\right)
$$

So, we have $M_{s}(u, v)=0$, a contradiction. Therefore $u=v$. The converse is obvious.

In the following theorem, we omit the continuity assumption of $f, g, T$ and $S$ and replace the compatibility of the pairs $(f, S)$ and $(g, T)$ by weak compatibility of the pairs, and we show that $f, g, S$ and $T$ have a common fixed point on $X$.

Theorem 2.2 Let $\left(X, b_{d}, \preceq\right)$ be an ordered complete $b$-dislocated metric space, and $f, g, S$ and $T$ be four self-maps on $X$ such that $(f, g)$ and $(S, T)$ are dominated and dominating maps, respectively, with $f X \subseteq T X$ and $g X \subseteq S X$, and $T X$ and $S X$ are $b_{d}$-closed subsets of $X$. Suppose that for all two comparable elements $x, y \in X$,

$$
\psi\left(2 s^{4} d(f x, g y)\right) \leq \psi\left(M_{s}(x, y)\right)-\varphi\left(M_{s}(x, y)\right)
$$

is satisfied, where

$$
M_{s}(x, y)=\max \left\{b_{d}(S x, T y), b_{d}(f x, S x), b_{d}(g y, T y), \frac{b_{d}(S x, g y)+b_{d}(f x, T y)}{4 s}\right\},
$$

$\psi \in \Psi$ and $\varphi \in \Phi$. Iffor every non-increasing sequence $\left\{x_{n}\right\}$ and a sequence $\left\{y_{n}\right\}$ with $y_{n} \preceq$ $x_{n}$, for all $n$ such that $y_{n} \rightarrow u$, we have $u \preceq x_{n}$, and the pairs $(f, S)$ and $(g, T)$ are weakly compatible, then $f, g, S$ and $T$ have a common fixed point. Moreover, the set of common fixed points off, $g, S$ and $T$ is well ordered if and only if $f, g, S$ and $T$ have one and only one common fixed point.

Proof Following the proof of Theorem 2.1, there exists $y \in X$ such that

$$
\lim _{k \rightarrow \infty} b_{d}\left(y_{k}, y\right)=0
$$

Since $T(X)$ is $b_{d}$-closed and $\left\{y_{2 n+1}\right\} \subseteq T(X)$, therefore $y \in T(X)$. Hence, there exists $u \in X$ such that $y=T u$ and

$$
\lim _{n \rightarrow \infty} b_{d}\left(y_{2 n+1}, T u\right)=\lim _{n \rightarrow \infty} b_{d}\left(T x_{2 n+1}, T u\right)=0 .
$$

Similarly, there exists $v \in X$ such that $y=T u=S v$ and

$$
\lim _{n \rightarrow \infty} b_{d}\left(y_{2 n}, S v\right)=\lim _{n \rightarrow \infty} b_{d}\left(S x_{2 n}, S v\right)=0
$$

Now we prove that $v$ is a coincidence point of $f$ and $S$.

Since $T x_{2 n+1} \rightarrow y=S v$ as $n \rightarrow \infty$, so, by assumption, $T x_{2 n+1} \preceq S v$. Therefore, from (2.23) we have

$$
\psi\left(2 s^{4} b_{d}\left(f v, g x_{2 n+1}\right)\right) \leq \psi\left(M_{s}\left(\nu, x_{2 n+1}\right)\right)-\varphi\left(M_{s}\left(\nu, x_{2 n+1}\right)\right),
$$


where

$$
\begin{aligned}
M_{s}\left(v, x_{2 n+1}\right)= & \max \left\{b_{d}\left(S v, T x_{2 n+1}\right), b_{d}(f v, S v), b_{d}\left(g x_{2 n+1}, T x_{2 n+1}\right),\right. \\
& \left.\frac{b_{d}\left(S v, g x_{2 n+1}\right)+b_{d}\left(f v, T x_{2 n+1}\right)}{4 s}\right\} \\
= & \max \left\{b_{d}\left(T u, T x_{2 n+1}\right), b_{d}(f v, y), b_{d}\left(g x_{2 n+1}, T x_{2 n+1}\right),\right. \\
& \left.\frac{b_{d}\left(S v, y_{2 n+2}\right)+b_{d}\left(f v, T x_{2 n+1}\right)}{4 s}\right\} .
\end{aligned}
$$

Taking the upper limit as $n \rightarrow \infty$ and using (2.25)-(2.26) and Lemma 1.32, we obtain that

$$
\begin{aligned}
& \max \left\{b_{d}(f v, y), \frac{1}{s^{2}} b_{d}(y, y), \frac{1}{4 s^{2}} b_{d}(y, y)\right\} \\
& \quad \leq \liminf _{n \rightarrow \infty} M_{s}\left(v, x_{2 n+1}\right) \\
& \quad \leq \limsup _{n \rightarrow \infty} M_{s}\left(v, x_{2 n+1}\right) \\
& \quad \leq \max \left\{0, b_{d}(f v, y), s^{2} b_{d}(y, y), \frac{0+s^{2} b_{d}(f v, y)}{4 s}\right\} \\
& \quad \leq \max \left\{b_{d}(f v, y), 2 s^{2} b_{d}(f v, y)\right\} \\
& \quad=2 s^{2} b_{d}(f v, y) .
\end{aligned}
$$

Taking the upper limit as $n \rightarrow \infty$ in (2.27) and using (2.28) and Lemma 1.32, we obtain that

$$
\begin{aligned}
\psi\left(2 s^{3} d(f v, y)\right) & =\psi\left(2 s^{4} \frac{1}{s} d(f v, y)\right) \\
& \leq \psi\left(2 s^{2} b_{d}(f v, y)\right)-\varphi\left(\liminf _{n \rightarrow \infty} M_{s}\left(v, x_{2 n+1}\right)\right) \\
& \leq \psi\left(2 s^{3} b_{d}(f v, y)\right)-\varphi\left(\liminf _{n \rightarrow \infty} M_{s}\left(v, x_{2 n+1}\right)\right),
\end{aligned}
$$

which implies that $\liminf _{n \rightarrow \infty} M_{s}\left(v, x_{2 n+1}\right)=0$, so from (2.28) we obtain $f v=y=S v$.

As $f$ and $S$ are weakly compatible, we have $f y=f S v=S f v=S y$. Thus, $y$ is a coincidence point of $f$ and $S$.

Similarly, it can be shown that $y$ is a coincidence point of the pair $(g, T)$. Now, we show that $f y=g y$. From (2.23) we have

$$
\psi\left(2 s^{4} d(f y, g y)\right) \leq \psi\left(M_{s}(y, y)\right)-\varphi\left(M_{s}(y, y)\right),
$$

where

$$
\begin{aligned}
M_{s}(y, y) & =\max \left\{b_{d}(S y, T y), b_{d}(f y, S y), b_{d}(g y, T y), \frac{b_{d}(S y, g y)+b_{d}(f y, T y)}{4 s}\right\} \\
& =\max \left\{b_{d}(f y, g y), b_{d}(f y, f y), b_{d}(g y, g y), \frac{b_{d}(f y, g y)+b_{d}(f y, g y)}{4 s}\right\}
\end{aligned}
$$




$$
\begin{aligned}
& =\max \left\{b_{d}(f y, g y), b_{d}(f y, f y), b_{d}(g y, g y)\right\} \\
& \leq \max \left\{b_{d}(f y, g y), 2 s b_{d}(f y, g y), 2 s b_{d}(f y, g y)\right\} \\
& =2 s b_{d}(f y, g y) .
\end{aligned}
$$

So, we have

$$
\begin{aligned}
\psi\left(2 s^{4} d(f y, g y)\right) & \leq \psi\left(M_{s}(y, y)\right)-\varphi\left(M_{s}(y, y)\right) \\
& \leq \psi\left(2 s b_{d}(f y, g y)\right)-\varphi\left(M_{s}(y, y)\right) \\
& \leq \psi\left(2 s^{4} b_{d}(f y, g y)\right)-\varphi\left(M_{s}(y, y)\right),
\end{aligned}
$$

which implies that $M_{s}(y, y)=0$, so we have $f y=g y$. Therefore, $f y=g y=S y=T y$.

Now, similar to the proof of Theorem 2.1, indeed from (2.20)-(2.22), we have $g y=y$. Therefore, $f y=g y=S y=T y=y$, as required. The last conclusion follows similarly as in the proof of Theorem 2.1.

Now, we give an example to support our result.

Example 2.3 Let $X=[0, \infty)$ be equipped with the $b$-dislocated metric $b_{d}(x, y)=(x+y)^{2}$ where $s=2$ and suppose that ' $\preceq$ ' is the usual ordering $\leq$ on $X$. Obviously, $\left(X, b_{d}, \leq\right)$ is an ordered complete $b$-dislocated metric space. Let $f, g, S, T: X \rightarrow X$ be defined as

$$
\begin{aligned}
& f(x)=\ln \left(1+\frac{x}{4}\right), \quad g(x)=\ln \left(1+\frac{x}{5}\right), \\
& S(x)=e^{5 x}-1, \quad T(x)=e^{4 x}-1 .
\end{aligned}
$$

For each $x \in X$, we have $1+\frac{x}{4} \leq e^{x}$ and $1+\frac{x}{5} \leq e^{x}$, so $f(x)=\ln \left(1+\frac{x}{4}\right) \leq x, g(x)=\ln \left(1+\frac{x}{5}\right) \leq x$, $x \leq e^{5 x}-1=S(x)$ and $x \leq e^{4 x}-1=T(x)$. Thus, $f$ and $g$ are dominated and $T$ and $S$ are dominating with $f(X)=g(X)=S(X)=T(X)=[0, \infty)$. Also, the pair $(g, T)$ is compatible, $g$ is continuous and $(f, S)$ is weakly compatible. Let the control functions $\psi, \varphi:[0, \infty) \rightarrow$ $[0, \infty)$ be defined as $\psi(t)=b t$ and $\varphi(t)=(b-1) t$, for all $t \in[0, \infty)$, where $1<b \leq \frac{400}{32}$. Note that

$$
\begin{aligned}
\psi\left(2 s^{4} b_{d}(f(x), g(y))\right) & =32 b(f(x)+g(y))^{2} \\
& =32 b\left(\ln \left(1+\frac{x}{4}\right)+\ln \left(1+\frac{y}{5}\right)\right)^{2} \\
& \leq 32 b\left(\frac{x}{4}+\frac{y}{5}\right)^{2}=\frac{32}{400} b(5 x+4 y)^{2} \\
& \leq\left(e^{5 x}-1+e^{4 y}-1\right)^{2} \\
& =b_{d}(S(x), T(y)) \\
& \leq M_{2}(x, y)=\psi\left(M_{2}(x, y)\right)-\varphi\left(M_{2}(x, y)\right), \quad x, y \in X .
\end{aligned}
$$

Thus, $f, g, S$ and $T$ satisfy all the conditions of Theorem 2.1. Moreover, 0 is a unique common fixed point of $f, g, S$ and $T$. 
Corollary 2.4 Let $\left(X, b_{d}, \preceq\right)$ be an ordered complete b-dislocated metric space, and let $f$ and $g$ be two dominated self-maps on X. Suppose that for every two comparable elements $x, y \in X$,

$$
\psi\left(2 s^{4} b_{d}(f x, g y)\right) \leq \psi\left(M_{s}(x, y)\right)-\varphi\left(M_{s}(x, y)\right)
$$

is satisfied, where

$$
M_{s}(x, y)=\max \left\{b_{d}(x, y), b_{d}(f x, x), b_{d}(g y, y), \frac{b_{d}(x, g y)+b_{d}(f x, y)}{4 s}\right\},
$$

$\psi \in \Psi$ and $\varphi \in \Phi$.Iffor every non-increasing sequence $\left\{x_{n}\right\}$ and a sequence $\left\{y_{n}\right\}$ with $y_{n} \preceq$ $x_{n}$, for all $n$ such that $y_{n} \rightarrow u$, we have $u \leq x_{n}$, then $f$ and $g$ have a common fixed point. Moreover, the set of common fixed points of $f$ and $g$ is well ordered if and only iff and $g$ have one and only one common fixed point.

Proof Taking $S$ and $T$ as identity maps on $X$, the result follows from Theorem 2.2.

Corollary 2.5 Let $\left(X, b_{d}, \preceq\right)$ be an ordered complete b-dislocated metric space. Let $f$ and $g$ be dominated self-maps on $X$. Suppose that for every two comparable elements $x, y \in X$,

$$
2 s^{4} b_{d}(f x, g y) \leq M_{s}(x, y)-\varphi\left(M_{s}(x, y)\right)
$$

is satisfied, where

$$
M_{s}(x, y)=\max \left\{b_{d}(x, y), b_{d}(f x, x), b_{d}(g y, y), \frac{b_{d}(x, g y)+b_{d}(f x, y)}{4 s}\right\},
$$

and $\varphi \in \Phi$. If for every non-increasing sequence $\left\{x_{n}\right\}$ and a sequence $\left\{y_{n}\right\}$ with $y_{n} \preceq x_{n}$, for all $n$ such that $y_{n} \rightarrow u$, it implies that $u \preceq x_{n}$, then $f$ and $g$ have a common fixed point. Moreover, the set of common fixed points off and $g$ is well ordered if and only iff and $g$ have one and only one common fixed point.

Proof If we take $S$ and $T$ as the identity maps on $X$ and $\psi(t)=t$ for all $t \in[0, \infty)$, then from Theorem 2.2 it follows that $f$ and $g$ have a common fixed point.

Remark 2.6 As corollaries we can state partial metric space as well as $b$-metric space versions of our proved results in a similar way, which extends recent results in these settings.

\section{Existence of a common solution for a system of integral equations}

Consider the following system of integral equations:

$$
\begin{aligned}
& x(t)=\int_{a}^{b} K_{1}(t, r, x(r)) d r, \\
& x(t)=\int_{a}^{b} K_{2}(t, r, x(r)) d r,
\end{aligned}
$$

where $b>a \geq 0$. The purpose of this section is to present an existence theorem for a solution to (3.1) that belongs to $X=C[a, b]$ (the set of continuous real functions defined on $[a, b]$ ) by using the obtained result in Corollary 2.4. 
Here, $K_{1}, K_{2}:[a, b] \times[a, b] \times R \rightarrow R$. The considered problem can be reformulated in the following manner.

Let $f, g: X \rightarrow X$ be the mappings defined by

$$
\begin{aligned}
& f x(t)=\int_{a}^{b} K_{1}(t, r, x(r)) d r \\
& g x(t)=\int_{a}^{b} K_{2}(t, r, x(r)) d r
\end{aligned}
$$

for all $x \in X$ and for all $t \in[a, b]$.

Then the existence of a solution to (3.1) is equivalent to the existence of a common fixed point of $f$ and $g$. According to Example 1.11, $X$ equipped with

$$
b_{d}(u, v)=\max _{t \in[a, b]}(|u(t)|+|v(t)|)^{p}
$$

for all $u, v \in X$, is a complete $b$-dislocated metric space with $s=2^{p-1}$.

We endow $X$ with the partial ordering $\preceq$ given by

$$
x \preceq y \quad \Longleftrightarrow \quad x(t) \leq y(t)
$$

for all $t \in[a, b]$. Moreover, in [4], it is proved that $(X, \preceq)$ is regular.

Now, we will prove the following result.

Theorem 3.1 Suppose that the following hypotheses hold:

(i) $K_{1}, K_{2}:[a, b] \times[a, b] \times R \rightarrow R$ are continuous;

(ii) for all $t, r \in[a, b]$ and $x \in X$, we have

$$
x(t) \leq \min \left\{\int_{a}^{b} K_{1}(t, r, x(r)) d r, \int_{a}^{b} K_{2}(t, r, x(r)) d r\right\} ;
$$

(iii) for all $r, t \in[a, b]$ and $x, y \in X$ with $x \preceq y$, we have

$$
\left(\left|K_{1}(t, r, x(r))\right|+\left|K_{2}(t, r, y(r))\right|\right) \leq \xi(t, r) \ln \left(1+(|x(r)|+|y(r)|)^{p}\right),
$$

where $\xi$ is a continuous function satisfying

$$
\sup _{t \in[a, b]}\left(\int_{a}^{b} \xi(t, r)^{p} d r\right)<\frac{1}{2^{4 p^{2}-3 p}(b-a)^{p-1}} .
$$

Then the integral equations (3.1) have a common solution $x \in X$.

Proof From condition (ii), $f$ and $g$ are dominated self-maps on $X$.

Let $1 \leq p, q<\infty$ with $\frac{1}{p}+\frac{1}{q}=1$. 
Now, let $x, y \in X$ be such that $x \succeq y$. From condition (iii), for all $t \in[a, b]$, we have

$$
\begin{aligned}
\left(2^{4 p-3}(|f x(t)|+|g y(t)|)\right)^{p} \\
\quad \leq 2^{4 p^{2}-3 p}\left(\int_{a}^{b}\left(\left|K_{1}(t, r, x(r))\right|+\left|K_{2}(t, r, x(r))\right|\right) d r\right)^{p} \\
\quad \leq 2^{4 p^{2}-3 p}\left[\left(\int_{a}^{b} 1^{q} d r\right)^{\frac{1}{q}}\left(\int_{a}^{b}\left(\left|K_{1}(t, r, x(r))\right|+\left|K_{2}(t, r, x(r))\right|\right)^{p} d r\right)^{\frac{1}{p}}\right]^{p} \\
\quad \leq 2^{4 p^{2}-3 p}(b-a)^{\frac{p}{q}}\left(\int_{a}^{b} \xi(t, r)^{p}\left(\ln \left(1+(|x(r)|+|y(r)|)^{p}\right)\right)^{p} d r\right) \\
\quad \leq 2^{4 p^{2}-3 p}(b-a)^{\frac{p}{q}}\left(\int_{a}^{b} \xi(t, r)^{p}\left(\ln \left(1+b_{d}(x, y)\right)\right)^{p} d r\right) \\
\quad \leq 2^{4 p^{2}-3 p}(b-a)^{\frac{p}{q}}\left(\int_{a}^{b} \xi(t, r)^{p}\left(\ln \left(1+M_{s}(x, y)\right)\right)^{p} d r\right) \\
\quad=2^{4 p^{2}-3 p}(b-a)^{p-1}\left(\int_{a}^{b} \xi(t, r)^{p} d r\right)\left(\ln \left(1+M_{s}(x, y)\right)\right)^{p} \\
<\left(\ln \left(1+M_{s}(x, y)\right)\right)^{p} \\
=M_{s}(x, y)^{p}-\left(M_{s}(x, y)^{p}-\left(\ln \left(1+M_{s}(x, y)\right)\right)^{p}\right) .
\end{aligned}
$$

Hence,

$$
\begin{aligned}
\left(2 s^{4} b_{d}(f x, g y)\right)^{p} & =2 s^{4} \sup _{t \in[a, b]}(|f x(t)|+|g y(t)|)^{p} \\
& \leq M_{s}(x, y)^{p}-\left(M_{s}(x, y)^{p}-\left(\ln \left(1+M_{s}(x, y)\right)\right)^{p}\right) .
\end{aligned}
$$

Taking $\psi(t)=t^{p}$ and $\varphi(t)=t^{p}-(\ln (1+t))^{p}$ in Corollary 2.4, there exists $x \in X$, a common fixed point of $f$ and $g$, that is, $x$ is a solution for (3.1).

\section{Competing interests}

The authors declare that they have no competing interests.

\section{Authors' contributions}

All authors contributed equally and significantly in writing this article. All authors read and approved the final manuscript.

\section{Author details}

${ }^{1}$ Department of Mathematics, King Abdulaziz University, P.O. Box 80203, Jeddah, 21589, Saudi Arabia. ${ }^{2}$ Department of Mathematics, Qaemshahr Branch, Islamic Azad University, Qaemshahr, Iran. ${ }^{3}$ Young Researchers and Elite Club, Kermanshah Branch, Islamic Azad University, Kermanshah, Iran. ${ }^{4}$ Department of Mathematics and Applied Mathematics, University of Pretoria, Lynwood road, Pretoria 0002, South Africa.

\section{Acknowledgements}

This article was funded by the Deanship of Scientific Research (DSR), King Abdulaziz University, Jeddah. Therefore, the first author acknowledges with thanks DSR, KAU for financial support.

Received: 6 July 2013 Accepted: 13 September 2013 Published: 07 Nov 2013

\section{References}

1. Aghajani, A, Abbas, M, Roshan, JR: Common fixed point of generalized weak contractive mappings in partially ordered b-metric spaces. Math. Slovaca (2012, in press)

2. Kelle, JL: General Topology. Van Nostrand, New York (1960)

3. Jungck, G: Compatible mappings and common fixed points. Int. J. Math. Math. Sci. 9(4), 771-779 (1986)

4. Nieto, JJ, Rodríquez-López, R: Existence and uniqueness of fixed points in partially ordered sets and applications to ordinary differential equations. Acta Math. Sin. Engl. Ser. 23, 2205-2212 (2007) 
5. Abbas, M, Nemeth, SZ: Finding solutions of implicit complementarity problems by isotonicity of metric projection. Nonlinear Anal. 75, 2349-2361 (2012)

6. Hussain, N, Kadelburg, Z, Radenović, S, Al-Solamy, FR: Comparison functions and fixed point results in partial metric spaces. Abstr. Appl. Anal. 2012, Article ID 605781 (2012)

7. Hussain, N, Đorić, D, Kadelburg, Z, Radenović, S: Suzuki-type fixed point results in metric type spaces. Fixed Point Theory Appl. 2012, 126 (2012)

8. Shah, MH, Simic, S, Hussain, N, Sretenovic, A, Radenovic, S: Common fixed points theorems for occasionally weakly compatible pairs on cone metric type spaces. J. Comput. Anal. Appl. 14, 290-297 (2012)

9. Hussain, N, Shah, MH: KKM mappings in cone b-metric spaces. Comput. Math. Appl. 62, 1677-1684 (2011)

10. Khamsi, MA, Hussain, N: KKM mappings in metric type spaces. Nonlinear Anal. 73, 3123-3129 (2010)

11. Hitzler, P, Seda, AK: Dislocated topologies. J. Electr. Eng. 51(12), 3-7 (2000)

12. Hitzler, P: Generalized metrics and topology in logic programming semantics. PhD thesis, National University of Ireland (University College, Cork) (2001)

13. Aage, CT, Salunke, JN: The results on fixed points in dislocated and dislocated quasi-metric space. Appl. Math. Sci. 2(59), 2941-2948 (2008)

14. Isufati, A: Fixed point theorems in dislocated quasi-metric space. Appl. Math. Sci. 4(5), 217-233 (2010)

15. Sarma, IR, Kumari, PS: On dislocated metric spaces. Int. J. Math. Arch. 3(1), $72-77$ (2012)

16. Shrivastava, R, Ansari, ZK, Sharma, M: Some results on fixed points in dislocated and dislocated quasi-metric spaces. J. Adv. Stud. Topol. 3(1), 25-31 (2012)

17. Shrivastava, M, Qureshi, K, Singh, AD: A fixed point theorem for continuous mapping in dislocated quasi-metric spaces. Int. J. Theor. Appl. Sci. 4(1), 39-40 (2012)

18. Kumari, PS, Kumar, VV, Sarma, IR: Common fixed point theorems on weakly compatible maps on dislocated metric spaces. Math. Sci. 6, 71 (2012)

19. Kumari, PS: Some fixed point theorems in generalized dislocated metric spaces. Math. Theory Model. 1(4), 16-22 (2011)

20. Daheriya, $\mathrm{RD}$, Jain, $\mathrm{R}$, Ughade, $\mathrm{M}$ : Some fixed point theorem for expansive type mapping in dislocated metric space. ISRN Math. Anal. 2012, Article ID 376832 (2012)

21. George, R: Cyclic contractions and fixed points in dislocated metric spaces. Int. J. Math. Anal. 7(9), 403-411 (2013)

22. Jha, K: A common fixed point theorem in dislocated metric space. Appl. Math. Sci. 6(91), 4497-4503 (2012)

23. Zoto, K: Some new results in dislocated and dislocated quasi-metric spaces. Appl. Math. Sci. 6(71), 3519-3526 (2012)

10.1186/1029-242X-2013-486

Cite this article as: Hussain et al.: Common fixed point results for weak contractive mappings in ordered $b$-dislocated metric spaces with applications. Journal of Inequalities and Applications 2013, 2013:486

\section{Submit your manuscript to a SpringerOpen ${ }^{\circ}$ journal and benefit from:}

- Convenient online submission

Rigorous peer review

- Immediate publication on acceptance

- Open access: articles freely available online

- High visibility within the field

- Retaining the copyright to your article 